\title{
De la phénoménologie de la religion à l'éthanalyse du fait juif
}

Jean-Michel Salanskis

\section{(2) OpenEdition}

1 Journals

Édition électronique

URL : https://journals.openedition.org/alter/2249

DOI : $10.4000 /$ alter.2249

ISSN : 2558-7927

Éditeur :

Association ALTER, Archives Husserl (CNRS-UMR 8547)

\section{Édition imprimée}

Date de publication : 1 novembre 2020

Pagination : 263-275

ISBN : 978-2-9550449-6-4

ISSN : $1249-8947$

\section{Référence électronique}

Jean-Michel Salanskis, « De la phénoménologie de la religion à l'éthanalyse du fait juif », Alter [En ligne], 28 | 2020, mis en ligne le 22 décembre 2020, consulté le 12 décembre 2021. URL : http://

journals.openedition.org/alter/2249; DOI : https://doi.org/10.4000/alter.2249

Ce document a été généré automatiquement le 12 décembre 2021.

Revue Alter 


\title{
De la phénoménologie de la religion à l'éthanalyse du fait juif
}

\author{
Jean-Michel Salanskis
}

1 Le thème "phénoménologie et religion" me pose doublement problème.

2 1) D'un côté l'orientation phénoménologique est quelque chose à quoi j'ai eu l'impression d'adhérer longtemps, par quoi je me sentais identifié même. Pourtant, je m'en sens de plus en plus séparé, par un indéfinissable écart stylistique au moins.

3 De plus, je n'ai jamais accepté le virage à une phénoménologie de l'être-au-monde, qui me semble dominer l'espace français pour y fixer le visage et l'identité de ce que l'on tient pour phénoménologie.

4 À vrai dire, le régime philosophique auquel j'aspire et dans lequel je me coule spontanément est un régime "subjectif" mettant en avant l'expérience - à l'instar du régime husserlien - mais divergeant d'avec ce précédent : ne serait-ce que parce que je suis influencé par l'esprit de mise à plat et la volonté déflationniste introduits par la philosophie analytique.

5 2) D'un second côté, ce qui m'intéresse dans le champ de ce que l'on regarde usuellement comme religieux, c'est le judaïsme. Mais précisément la compréhension que je défends depuis 2003 consiste à ne pas classer ce dernier comme religieux, à refuser le label de religion pour le judaïsme.

6 Cela étant dit, ces deux difficultés, regardées "dans l'autre sens" en quelque sorte, témoignent de mon rattachement à la thématique soulevée par la revue Alter. Je ne peux pas dire que le moment méthodologique de la phénoménologie n'ait pas compté et ne continue pas de compter en tant que strate formatrice pour moi, je ne peux pas nier que mon travail sur le judaïsme ne manifeste un refus de la morgue agnostique, critique et rationaliste devant tout ce qui se classe dans le religieux.

7 Pour aller plus avant, pourquoi ne pas commencer par tenter un éclaircissement de la notion de religion? 


\section{La notion de religion} christianisme : c'est le cas culturellement le plus familier (y compris pour les adeptes d'une autre religion). Le concept de religion émane donc pour nous d'une de ses instances, selon un procédé logique plus fréquent qu'on ne veut bien le reconnaître. Selon un tel concept, une religion est essentiellement :

9 - Fondée sur la thèse ontologique d'un Dieu maître et créateur de l'être. Pour presque tout interlocuteur de toute discussion, si l'on n'a pas réussi à établir l'existence d'une telle entité, le message religieux se dissout et perd toute crédibilité.

10 - Porteuse de la bonne nouvelle d'une vie éternelle après la mort : la religion nous recrute en nous donnant ce qu'aucune autre instance ne peut nous donner. Elle s'adresse à notre vœu de bonheur.

11 - Exprimée dans une disposition ou une propriété psychologique appelée foi, qui est l'élément subjectif corrélatif de la thèse théiste. Si l'on n'est pas capable de la foi, on ne saurait professer une religion.

12 Bien sûr, ce schéma est discutable, il est par trop fonction du cas qu'il relève au plan conceptuel. Pour le critiquer, il faudrait une compétence en matière de religions à laquelle je ne prétends pas. Remarquons cependant:

- Que ce schéma ne couvre pas parfaitement toutes les manières de vivre le christianisme luimême (certains l'abordent d'une manière existentielle, ou différemment sociale, et dans les deux cas les trois paramètres se trouvent relativisés).

- Les trois paramètres conviennent surtout au christianisme et à l'islam. Le schéma ne permet guère de comprendre l'indouisme, le bouddhisme ou les religions africaines.

Nous pouvons néanmoins nous demander comment se pose le problème d'une objectivité "religieuse" réclamant une "constitution phénoménologique" si l'on adopte le schéma spécifié par nos trois paramètres. Quelques réponses se suggèrent spontanément :

- Réfléchissant sur la thèse théiste, on se demandera en phénoménologue si l'on peut définir un profil phénoménal de Dieu, envisager celui-ci comme une objectivité à constituer. Et si la réponse est positive, a-t-on trahi le théologisme en lui donnant un tel enracinement?

- Réfléchissant sur la promesse de la vie immortelle, on peut se demander si nous savons faire sens d'une telle notion : l'esprit phénoménologique ne nous enseigne-t-il pas que l'ajout de l'immortalité fait sortir de l'eidos de la vie? Ce qui semblerait vouloir dire que la promesse en question n'a jamais de sens pour nous en fin de compte.

- Réfléchissant sur la foi, on peut essayer de la traduire comme un mode intentionnel tourné vers Dieu, l'appréhendant. Pourtant, il y a toute une tradition d'argumentation réalisterationaliste en faveur de l'existence de Dieu refusant d'entrer dans le pathétique de la foi : chacun a en tête la preuve ontologique ou la preuve physico-cosmologique, et il faudrait ajouter des propositions comme les récents arguments de logique modale d'un Plantinga ${ }^{1}$. Penser la religion à l'aune d'une phénoménologie existentielle de la foi, n'est-ce pas faire tort à une moitié de la tradition théologique? 


\section{De la phénoménologie à l'ethanalyse} précis sur la distance qui est aujourd'hui la mienne à l'égard de l'entreprise de la phénoménologie. Elle se traduit par le développement, programmé en 2001 et engagé en 2007, d'un nouveau régime philosophique, auquel j'ai donné le nom d'ethanalyse.

Il est indéniable que j'ai d'abord conçu un tel aggiornamento comme interne à la phénoménologie : il s'agissait seulement, pensais-je, de faire passer celle-ci au plan intersubjectif, à la fois pour ce qui regarde le constitué et pour ce qui regarde le constituant (j'ai formulé les choses ainsi dans un ouvrage jamais publié écrit au début des années 1980, dont le but était de conquérir une phénoménologie du politique).

Dans un second temps, j'ai perçu ce que je désirais comme une radicalisation de la révolution kantienne. Cette dernière, à mes yeux, consiste dans la spécialisation de la philosophie à l'étude du normatif partagé. La philosophie cesse de prétendre à une posture de connaissance "en plus" de toutes les connaissances, visant les mêmes objets que les savoir qu'elle couronne, pour devenir la méta-connaissance de ce qui fait légitimité pour nous. Ce que je viens de dire reformule l'abandon de la métaphysique dogmatique et l'orientation transcendantale.

Un tel déplacement est pour moi lié aussi à la leçon apprise chez Levinas : une leçon qui concerne la "strate humaine" cette fois. L'humain pour un regard lévinassien ne se comprend pas au niveau factuel et positif, on ne peut pas se limiter à le cerner dans le langage de l'ontologie. Le plus humain de l'humain, en effet, réside des les horizons normatifs avec lesquels nous vivons, qui transfigurent pour nous à la fois les positivités que nous fréquentons et les positivités que nous sommes.

Ma démarche, à vrai dire, a eu comme première étape la proposition d'une nouvelle "compréhension du sens". Il s'agissait pour moi de divorcer d'avec ce que je ressentais comme une conception du sens partagée par le courant phénoménologique et par le courant analytique : le sens est sens d'un objet, et il s'interprète à vrai dire comme le mode de présentation de cet objet ou le mode d'accès à cet objet. On peut éclairer cela par deux diagrammes résumant les approches de Frege et de Husserl : 


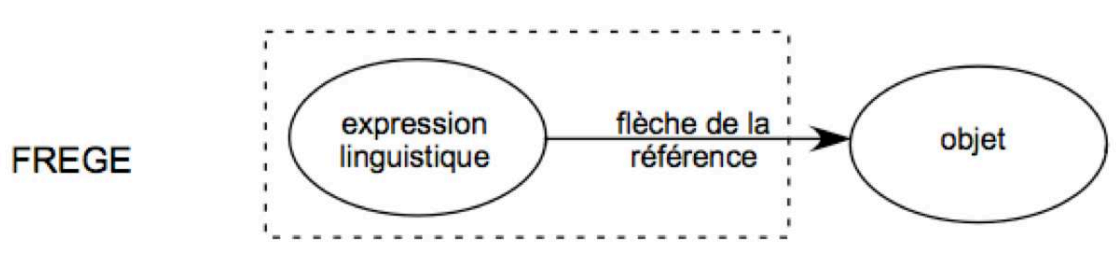

sens = expression linguistique transfigurée par la flèche référentielle

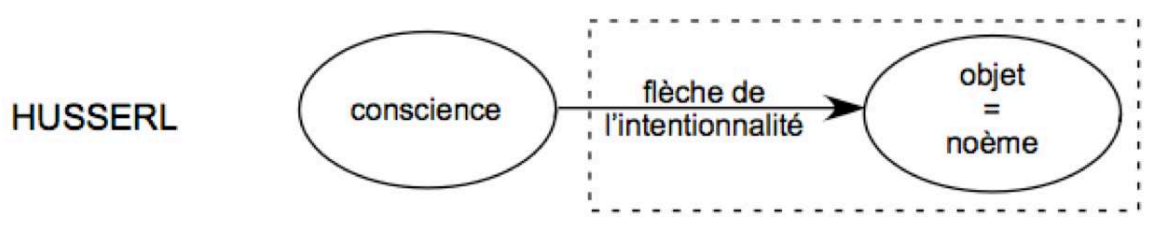

sens = objet transfiguré par la flèche intentionnelle

Chez Frege, un sens est porté par une expression linguistique (l'élève de Platon) qui détermine un mode de présentation de sa déno-tation (Aristote). Le sens est donc l'expression linguistique transfigurée par la flèche référentielle vers sa dénotation.

Chez Husserl, le sens est l'intentionné comme tel : mon objet c'est-à-dire mon noème en tant que visé par une certaine configuration (morphè) intentionnelle. L'objet transfiguré par la flèche intentionnelle vers lui.

Dans les deux cas, la "transfiguration" met au premier plan le mode de la présentation.

À cette conception aux deux visages j'oppose la vision post-lévinassienne du sens, à laquelle correspond un nouveau diagramme envisageant le sens à l'aune de l'atteinte et de la relance :

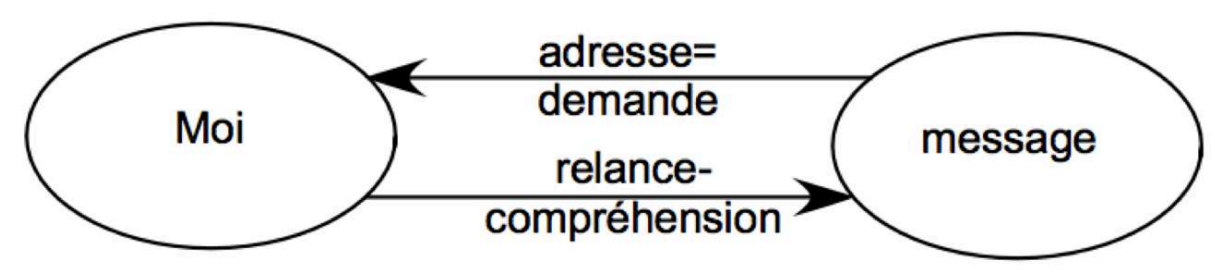

La circonstance directrice du sens, c'est qu'un message m'atteint : j'éprouve qu'il me demande que je le comprenne conformément à la directive qu'il est, c'est-à-dire que je le relance d'une manière qui en témoigne. Ce diagramme n'enveloppe aucune présupposition ontologique sur le destinateur, seul compte le déontologique de la demande et de l'atteinte.

4 Si l'on appréhende le sens de cette manière, partager un sens, ce sera partager la circonstance directrice, c'est-à-dire la réquisition. Au lieu que nous regardions la même chose suivant le même filtre, comme chez Frege ou Husserl, nous vivons une coréquisition : le même appel arrive auprès de chacun(e) de nous. 
comprendrions rien à la notion d'appel, et ne pourrions pas nous envisager nousmêmes comme ayant à observer les commandements de la sémance si nous n'appartenions pas à l'humanité de l'obligation, de la demande et de la prescription. Levinas enseigne que nous entrons dans cette sphère déontologique à partir de l'intrigue éthique : devant le visage, nous apprenons simultanément la signification de la moralité et qu'autrui nous incombe, qu'il nous est commandé d'être son gardien; du même coup, nous apprenons également l'être-obligé, nous entrons dans le plan déontologique de l'affaire humaine. Levinas situe un tel événement dans une étrange anthropologie déontologique qui est la sienne : l'humain est regardé par lui à l'aune de son intolérance envers l'absurdité positive-processuelle de l'être. Le plus humain de l'humain est aux yeux de Levinas le vœu d'évasion qui s'exprime au sein même de notre expérience de la brutalité nue de l'être et de son englobement. Et c'est ce vœu d'évasion qui accomplit son destin dans l'intrigue éthique, y trouvant une échappatoire à la "logique de l'être".

30 Je peux reprendre l'enseignement lévinassien dans le langage de l'ethanalyse, et dire que tout se passe, pour Levinas, comme s'il y avait un “archi-ethos", présupposé par tous les ethos, parce qu'il délivre la clef ultime de l'épreuve de la demande, du commandement. Archi-ethos dont le sollicitant serait le mot humain. Cet archi-ethos 
posséderait une sémance composée, essentiellement, du vœu d'évasion et du commandement du visage, c'est-à-dire qu'il ne serait pas purement prescriptif (incluant un désir en plus de son commandement fondamental).

31 Cet archi-ethos, de plus, ne serait pas facultatif comme les autres, il serait "catégorique" au sens kantien. Non pas au sens où les humains seraient incapables de lui déroger - bien au contraire, ils ne font que cela, c'est leur tendance majeure - mais au sens où s'en exempter au plan du droit est se détourner de son humanitée ${ }^{\text {. }}$

Quel est alors le jeu des ethos et de l'archi-ethos? Bien que chaque ethos présuppose l'archi-ethos et lui doive son sens d'ethos, ainsi que nous venons de le voir, chaque ethos tend aussi à s'imposer comme l'unique référence déontologique, à faire écran au commandement éthique originaire de l'archi-ethos. On éprouve que l'on n'a pas d'autre loi à suivre que celle de l'amour ou celle de la vérité, tournant le dos au fond éthique de l'obligation.

Nos vies humaines se vivent donc dans une double perplexité :

1. D'un côté, à chaque instant, nous pouvons nous demander à quel ethos consacrer notre prochain comportement. La question se pose de savoir si notre urgence est celle de l'amant ou du savant, par exemple. À quelle sémance devons-nous avoir égard, dans quel registre devons-nous situer notre agir, nos paroles, nos vécus?

2. D'un autre côté, nous sommes fondés à nous demander, à chaque instant également, si nous sommes suffisamment fidèles à l'archi-ethos en suivant l'horizon infini qui nous est ouvert par tel ou tel ethos, dont nous sommes adeptes. N'avons-nous pas gravement méconnu l'exigence éthique en n'ayant égard qu'à l'amour et sa loi, ou qu'à la vérité et ses commandements?

34 On peut demander quelle est la méthode de l'investigation éthanalytique. La réponse est qu'une fois reconnu le sollicitant comme tel (tout commence par là, il s'agit d'avoir l'oreille pour un mot qui porte un appel), on atteint la sémance de l'ethos correspondant d'une part en cherchant en soi-même ce à quoi il faut tenir pour ne pas céder sur l'enjeu, d'autre part en déduisant de tels commandements du comportement des autres adeptes. Nous l'humanité avons la faculté étrange de lire dans les mots et actes des autres ce sur quoi ils se règlent. C'est ce que j'appelle empathie déontique.

\section{Le fait juif}

Étant donc arrivé à une telle version réaménagée de la phénoménologie, je me suis intéressé de façon plus précise à ce que j'appelle le fait juif.

Je désigne de ce nom quelque chose qui insiste et persiste dans l'histoire humaine, y laisse des traces, avec comme support pour une telle insistance un collectif, appelé en général peuple juif. Fait juif s'entend donc en un sens non positif et non empirique du fait: ce que j'appelle fait est ici le Faktum dans l'histoire d'une tradition qui arrive jusqu'à nous pour nous offrir un avenir (pour s'offrir un avenir à travers nous).

37 Ce qui persiste, je l'identifie principalement comme ce que j'appelle l'étude-observance (bien que son couple ne soit assumé la plupart du temps que par une minorité).

Pour mieux comprendre cette notion, il faut analyser le cheminement historique du fait juif suivant deux dimensions :

1. Il y a quelque chose qui s'appelle la loi juive, et qui est formulé et décidé au fil des siècles par des docteurs spécialisés dans l'étude de cette loi, jugés plus compétents que les autres (les 
possekim). La loi est ainsi élaborée, en vue de la pleine richesse et la pleine précision de ce qu'elle stipule, par une filiation historique d'intellectuels, chacun héritant du travail de ses prédécesseurs.

2. Il y a des personnes qui sont comptées par le système comme obligés par cette loi, comme ayant à l'observer (et à l'étudier, d'ailleurs, au niveau qui est le leur). Ce sont ces personnes qui sont désignées comme juives : soit elles le sont parce que leur mère l'était, soit elles le sont parce qu'elles l'ont solennellement choisi devant un tribunal dans la procédure dite de conversion (Guerout).

Le cheminement du fait juif consiste donc dans une production toujours renouvelée de la loi et de personnes sur qui elle tombe. Il faut à ce processus une origine. Selon le mythe il y a au départ un "docteur de la loi" originaire - à savoir Moïse - et un ensemble originaire de destinataires de cette loi, à savoir « ceux qui campent auprès du Sinaï », un ensemble qui regroupe selon la tradition les Juifs échappés d'Egypte et des personnes ayant choisi de partager leur aventure (on les appelle le "grand mélange").

L'histoire de la persistance juive est donc l'histoire conjointe du travail des docteurs sur la loi et de la fécondité des familles juives : tout repose sur l'hypothèse que les rejetons des familles continueront de regarder la loi comme une loi pour eux, ce qui ne va pas de soi.

Quel est, au plan intrinsèque, le lien de l'étude et de l'observance ? Il est triple :

1) À un premier niveau très simple, j'ai besoin d'étudier pour savoir exactement ce qu'il faut faire, ce que tel ou tel commandement de la loi requiert de moi. Cela peut être non immédiat et passer par des clauses subtiles, je démêle ce qu'il en est en étudiant.

3 2) À un second niveau, l'étude est incluse dans ce qui est commandé. L'observance enveloppe l'étude comme une partie de son contenu.

3) À un troisième niveau, l'étude intervient pour définir l'enjeu ultime de l'observance. C'est le Maharal de Prague qui a clarifié cela, en déclarant en substance que, dans l'idéal, chacun devrait non seulement observer les commandements, mais encore les observer en considérant le monde humain selon le système des concepts exprimant le point de vue de la loi, et en rendant le monde humain conforme à une telle grille à vrai dire. L'horizon de l'observance est donc celui d'une humanité vivant sa vie personnelle et collective au plan conceptuel, s'arrachant du plan inférieur et immédiat.

Le fait juif est l'expression, avec son degré de réussite et son degré d'échec, du projet de l'étude-observance : projet d'un collectif qui observerait la loi tout en l'étudiant, selon le schéma de solidarité de l'un et de l'autre qui vient d'être précisé.

Il faut en même temps le considérer avec un regard lévinassien. Le projet est motivé par le maximum éthique mis en lumière par Levinas : ce que je dois à mon frère ne saurait être limité par contrat, ne se laisse pas intégrer à ce qu'a planifié mon contrôle calculant ; par suite ma dédicace éthique envers autrui est illimitée, elle porte en elle une structure d'infinitisation. L'idée de la tradition juive serait alors que le collectif de l'étude-observance est un collectif de personnes vigilantes, réveillées et rendues attentives par le souci de la loi et de sa compréhension, et que cette vigilance est favorable à l'exercice de la dédicace éthique illimitée. Cette dernière serait, donc, la finalité ultime. Et l'articulation avec la loi va plus ou moins de soi : lorsqu'on étudie les attendus de la loi juive, on découvre généralement que celle-ci envisage l'enjeu de l'amélioration et de la réparation du monde humain de manière "infiniment" 
exigeante. La loi juive vise une justice radicale, et pas seulement un monde équilibré sur le plan pragmatique.

On interroge souvent le fait juif sous l'angle de son particularisme, dont on lui fait reproche. Ce particularisme est indéniable : l'horizon du fait juif est la réalisation de la justice au sein du laboratoire particulier du peuple juif. En même temps, les sages ont toujours pensé que le contenu de cette expérience était offert à l'humanité, que les autres peuples pouvaient y prendre inspiration, en adopter des éléments, en reprendre des formes. On a donc affaire à, comme le dit Georges Hansel, un "particulier à rayonnement universel"4.

Cela nous conduit à interpréter correctement la catégorie de l'élection. Pour la tradition juive, l'élection correspond au fait que seul le peuple juif hérite du fardeau de la loi juive. L'élection, c'est le fait que le non-juif n'est pas soumis aux lois de Shabbat par exemple: une réalité historico-sociale comparable au fait qu'une personne allemande faisant carrière dans la chose intellectuelle, même en France, n'est pas jugée devoir exciper de la qualité d'ancienn(e) élève d'une ENS ou d'agrégé(e). Cette mitsva est réservée aux personnes ayant grandi dans l'enclave hexagonale.

Si on élabore la vision juive de l'excellence individuelle, alors il faut rappeler que celleci est située dans l'ordre éthique, et que, selon l'adage traditionnel «Le juste des nations est l'égal du grand prêtre » : pour atteindre l'excellence individuelle, nul besoin d'en passer par la condition juive.

Reste qu'on peut envier comme une grandeur et une noblesse le fait d'avoir le fardeau de la loi juive sur ses épaules: une loi si exigeante, nous pourchassant dans chaque aspect de la vie. On le peut, mais cela veut dire en tout cas abandonner le référentiel le plus usuel de l'existence contemporaine, celui de la liberté et de sa créativité : depuis la seconde moitié du XXe siècle, ne juge-t-on pas le plus souvent que le but de nos vies est d'abriter une création artiste de soi et des valeurs, non limitée par aucun oukase et aucun reliquat culturel?

51 Si l'on est vraiment passé du côté de la valorisation de notre faculté d'observance et de responsabilité, alors on est un candidat parfait à la guerout ou conversion : il suffit de se mettre en quête d'un tribunal qui fera de vous un(e) nouvel(le) obligé(e) des commandements du judaïsme.

Levinas, par ailleurs, il convient sans doute de le rappeler à ce stade de l'exposition, soutient que - comme êtres humains - nous avons tous l'expérience de l'élection dans l'intrigue éthique. Devant le visage en effet j'apprends que je suis le gardien de mon frère, et je suis élu par une telle responsabilité : elle est indéclinable, je ne peux pas la décaler vers un autre porteur. C'est ce qui fait d'une telle responsabilité un fardeau. Mais en même temps comme élection, elle se laisse revendiquer comme une sorte de gloire (pas une gloire que je m'attribue selon Levinas, mais la gloire témoignant d'un pôle de transcendance). Nous connaissons ainsi l'élection non pas comme un avantage que nous jalousons, mais comme quelque chose qui nous accable.

Dernier point, peut-être le plus important: ce qui précède voulait manifester que la dimension religieuse du judaïsme est en quelque sorte facultative. On peut interpréter la loi comme ce qui vient d'un être innommable au-delà du monde, et vivre son adhésion à elle comme révérence envers cet être, mais c'est facultatif et de toute façon, pour savoir ce que veut la loi, on se tourne vers les enseignements des sages au fil des siècles, vers le travail humain de l'élaboration de la loi juive par ses docteurs (ce qui 
conduisait Georges Hansel à écrire que dans le judaïsme la révélation se décide à la majorités).

La foi n'est pas ce qui est demandé à la personne juive : c'est plutôt l'observance, le suivi concret des préceptes de la loi, et l'étude comme nous l'avons expliqué. Certains versets sont commentés dans le sens de l'assertion d'un monde futur ouvert ou offert au juste, mais cela ne signifie pas forcément le dogme de l'immortalité tel qu'il circule dans le christianisme. Le point important pour la tradition juive est de ne pas nier que cette tradition envisage plus que le fonctionnement social, s'adresse à l'enjeu du sens de l'histoire et du sens de la vie.

Il est donc possible de vivre une vie juive observante sans que cela passe par la thèse théologique, la catégorie psychologique de la foi ou l'attente de la récompense d'une vie éternelle (comme dit Levinas les consolations de la religion ne sont méritées que par ceux qui n'en ont pas besoin ${ }^{6}$ ). Et de telles attitudes existent. On comprend pourquoi Samson Raphaël Hirsch, un des pères de ce qu'on appelle le courant "moderneorthodoxe", professait que le judaïsme n'était pas une religion: il est plutôt l'organisation d'une vie individuelle et collective pour un groupe humain historique traversant l'histoire.

\section{Le fait juif et l'ethanalyse}

Dans ces conditions, me demandera-t-on, quelle est l'intersection ou l'interférence du judaïsme ainsi compris avec la post-phénoménologie de l'ethanalyse, décrite un peu plus haut dans ses grandes lignes?

Pour commencer, selon les analyses que j'ai proposées, il y a un ethos juif, qui constitue donc un cas particulier pour l'ethanalyse, à côté de ceux de l'amour et de la vérité, déjà évoqués. Je pourrais même ajouter ici que mon premier essai timide de mise en œuvre de l'ethanalyse portait sur cet ethos juif : telle était la tentative de mon essai de 2003 Extermination, loi, Israël ${ }^{7}$. Seulement dans cet ouvrage je n'avais pas encore compris moimême la nature des enquêtes éthanalytiques, en telle sorte que les conclusions formulées ne relèvent pas vraiment du genre défini ultérieurement.

Avec le recul, je peux formuler un certain nombre de propriétés spécifiques de l'ethos juif :

1. Le sollicitant est linguistiquement double: juif/Juif [l'adjectif et le substantif]. Cela correspond à la dualité entre la loi juive et les Juifs à qui elle prétend s'appliquer.

2. L'ethos juif, en plus du comportement particulier qu'il appelle, se manifeste comme gardien de la grandeur "absolue" ou "générale" de l'hétéro-affection ou de l'hétéronomie. Il enseigne à celles et ceux qui le comprennent la grandeur de l'élection, alors que celle-ci est plutôt décriée dans la société contemporaine. En ce sens l'ethos juif aide à comprendre tout ethos. D'ailleurs la description de notre partage humain comme "chapeauté" par les vectorisations infinies des ethos conduit à la vision d'une mosaïque de "judaïsmes" couronnant notre monde (chaque ethos est un judaïsme local).

3. L'ethos juif se veut au service de l'archi-ethos : l'étude-observance est supposée engendrer l'humain vigilant capable d'entendre l'appel éthique et de l'honorer.

4. L'ethos juif enveloppe l'étude et l'explicitation en interne de sa sémance : la tradition de la loi juive est exactement cela, elle formule tout ce qui est implicitement commandé dans «Comporte toi en Juif!» ou dans «Obéis à la loi juive! ». Il m'est arrivé dans le passé de suggérer que la littérature ou la psychanalyse avaient un statut comparable vis-à-vis de 
l'ethos de l'amour : en y réfléchissant mieux, je pense qu'il y a en l'espèce seulement une analogie imparfaite.

59 Au-delà de ces éléments, il est naturel de s'interroger sur le rapport qu'une telle démarche noue implicitement entre philosophie et judaïsme: pour qui écrit dans l'hexagone, impossible d'oublier le souci de la laïcité et de sa préservation !

Comme je l'ai déjà dit, l'ethanalyse, par un côté, utilise pour dépeindre la culture humaine quelque chose que le monde usuellement classe comme religieux : on la voit comme abritant, au niveau décisif de ce qui en elle est vectorisation et infinitisation, une mosaïque de judaïsmes. Le "religieux" fonctionne donc comme catégorie descriptive pour le monde laïque (mais il faut rappeler ici que le point de vue soutenu ici refuse en profondeur l'assimilation du judaïsme au modèle religieux).

61 D'un autre côté, l'ethanalyse classe le judaïsme comme un ethos. Donc elle le regarde comme un cas - particulier quoiqu'éminent - de ce que l'on pourrait appeler l'idéalisme culturel. Le judaïsme est une des manières de partager une orientation infinitaire, comparable en cela à l'amour et à la vérité.

62 Tels sont les résultats un peu étranges d'une démarche héritière de la phénoménologie, bien que désormais sensiblement décalée par rapport à elle, tirant une inspiration importante d'une très vieille tradition usuellement perçue comme religieuse, la tradition de la loi juive.

\section{NOTES}

1. Cf. R. Pouivet, Philosophie contemporaine, Paris, PUF, 2018, p. 120-124.

2. Son titre était Le politique : transcendance et identité de camp, et son contenu esquisse à vrai dire l'ethanalyse du politique proposée dans Territoires du sens (J.-M. Salanskis, Paris, Vrin, 2007).

3. Un tel geste est imaginé dans la série Vampire Diaries: à un moment de l'intrigue, Caroline donne congé à son humanité, et Stefan la suit par amour dans cette déchéance.

4. Cf. http://akadem.org/sommaire/paracha/5776/parachat-hachavoua-5776/le-h-le-ha-undouble-monotheisme-10-09-2015-73270_4611.php

5. Cf. G. Hansel, Explorations talmudiques, Paris, Éditions Odile Jacob, 1998, p. 33.

6. $C f$. « Toi qui me regardes », entretien avec France Guwy.

7. Cf. J.-M. Salanskis, Extermination, loi, Israël, Paris, Les Belles Lettres, 2003. 